\title{
Gygnus Loop and Some Related Nebulosities
}

\author{
R. MINKowSKI \\ Mount Wilson and Palomar Observatories, \\ Carnegie Institution of Washington, California Institute of Technology, Pasadena, California
}

$\mathbf{T}$ HE suggestion by Oort ${ }^{1,2}$ that the Cygnus loop, an object of a diameter of about $3^{\circ}$ whose brightest parts are NGC 6960 and NGC 6992, is an expanding supernova shell slowed down by interaction with the interstellar medium has led to an increased interest in objects of this type. As better information on the Cygnus loop becomes available, it seems to become increasingly probable that Oort's suggestion provides an acceptable interpretation.

Objects similar to the Cygnus loop are rare, but it is not unique. A very similar object is IC 443. Its appearance probably is best described as that of an incomplete thick shell. The inner boundary, with a diameter of about $33^{\prime}$, is brightest in the northeast, where it actually forms the brightest part of the nebula. The outer boundary is quite incomplete and visible only in the southeast quadrant; its diameter would seem to be about $1^{\circ}$. In both objects sharp filaments are very conspicuous features, but there is a background of more diffuse streaks and clouds. It seems very probable that the nebulosity found in the position of the radio source $\mathrm{HB} 9^{3}$ is another object of this type; the diameter of the nebula is about $2^{\circ}$, that of the radio source $1.4^{\circ}$. There are however only very few sharp filaments. Another object of similar type is in the position of the radio source HB 21. This nebulosity is in a very complicated area, near the edge of, but still within, the extended nebulosity in Cygnus. It has a size of about $2^{\circ} \times 2.5^{\circ}$, but it is tempting to assume that some features of the extended Cygnus nebulosity about $3^{\circ}$ southwest from the center are somehow affected by the nebulosity. The object contains only a few extremely faint rudimentary features of filamentary character. Finally, it seems possible that the large filamentary nebula $S 147^{4}$ is also a member of this group of objects. The largest diameter is about $4^{\circ}$ in the $\mathrm{E}-\mathrm{W}$ direction. The long-stretched filamentary structures which appear on an extremely faint diffuse background are mostly rather diffuse, but some filaments are quite sharp.

The first four of these objects are nonthermal radio sources of considerable flux density. The last one has formerly not been identified as a radio source, but the map of the flux densities for the Taurus region by

\footnotetext{
1 J. H. Oort, Monthly Notices Roy. Astron. Soc. 106, 159 (1946)

2 J. H. Oort, "Problems of cosmical aerodynamics," Central Air Documents Office, Dayton, p. 118 (1951).

${ }^{3}$ R. Hanbury Brown and C. Hazard, Monthly Notices Roy. Astron. Soc. 113, 123 (1953).

${ }^{4}$ V. F. Hase, and G. A. Shajn, Izvest. Crimean Astrophys. Obs. 7, 93 (1951); 9, 52 (1952).
}

Seeger, Westerhout, and van de Hulst ${ }^{5}$ shows a ridge of high flux density extending northward from the Crab Nebula which might be explained if $\mathrm{S} 147$ is a radio source of a flux density which, if the size of the object is taken into account, may be not much below that of IC 443.

If all five objects are of similar nature, the nonthermal radio emission is a common feature, but the formation of intense sharp filaments in great numbers does not seem to occur necessarily or perhaps not during all phases of the history of these objects.

The only one of these objects that has been investigated in some detail is the Cygnus loop. It is long known that the object is expanding. The edges of its brightest parts, NGC 6960 and NGC 6992, move outward. The value of the motion determined by $\mathrm{Hubble}^{6}$ is $0.03^{\prime \prime}$ per year; the age of $150000 \mathrm{yr}$, which would be obtained if the expansion had been uniform, is an upper limit. Radial velocities have now been determined for 25 points distributed over the object. The observations were carried out with the nebular spectrograph at the 200 -in. telescope, using a dispersion of $215 \mathrm{~A} / \mathrm{mm}$ for $\mathrm{H} \alpha$ and [NII] $\lambda 6548 / 6584$; the mean error is about $\pm 10 \mathrm{~km} / \mathrm{sec}$. The results are shown in Fig. 1 where the velocities have been plotted against the distance from the center of the loop, assumed to be at $(1950) 20^{h} 49.5^{m},+30^{\circ} 48.5^{\prime}$. No systematic difference seems to exist between the motions of the sharp filaments and the more diffuse features. Dashed lines connect values measured for different features appearing on one spectrogram obtained with a slit length of $30^{\prime \prime}$ The local velocity differences are large and not much smaller than the velocities. Similar scatter exists in the motions. If the one point obtained in the southern extension (at a distance of $124.5^{\prime}$ ) is disregarded, all points are in an area bordered by two ellipses. They define a thick shell with an inner diameter of about $80^{\prime}$ and an outer diameter of about $160^{\prime}$; the velocity of expansion is $46 \mathrm{~km} / \mathrm{sec}$ at the inner border, $116 \mathrm{~km} / \mathrm{sec}$ at the outer border which includes NGC 6960 and NGC 6992 . The velocity of the object as a whole is $+16 \mathrm{~km} / \mathrm{sec}$, or $+33 \mathrm{~km} / \mathrm{sec}$ relative to the local standard of rest, a velocity which is about $+25 \mathrm{~km} / \mathrm{sec}$ larger than expected as an effect of the galactic rotation. In view of the uncertainty which the

${ }^{5}$ Seeger, Westerhout, and van de Hulst, Bull. Astron. Soc. Neth. 13, 89 (1956).

${ }^{6}$ E. P. Hubhle, Carnegie Institution Yearbook, No. 36, p. 189 (1937). 
TABLE I. Intensities of the main lines in the Cygnus loop.

\begin{tabular}{|c|c|c|c|c|c|}
\hline Line & $\underset{\text { lain }}{\text { Chamber- }}$ & $\begin{array}{c}1953 \\
\text { skinkow- }\end{array}$ & Pikelner & Code & $\begin{array}{c}1957 \\
\text { Minkowski }\end{array}$ \\
\hline OII 3727 & 204 & 189 & 530 & 179 & $\cdots$ \\
\hline $\mathbf{H} \beta$ & $\cdots$ & 19 & 21 & 15 & 21 \\
\hline OIII $4959+5007$ & 100 & 100 & 100 & 100 & 100 \\
\hline $\begin{array}{l}\mathrm{H} \alpha \\
\text { NII } 6548+6584\end{array}$ & \} 36 & $\ldots$ & 132 & $\cdots$ & \} $128\left\{\begin{array}{l}72 \\
56\end{array}\right.$ \\
\hline
\end{tabular}

velocity picture obviously has, this difference cannot be considered as significant.

The over-all picture of the Cygnus loop suggested by the arrangement of the velocities is surprisingly similar to that of IC 443 . It is also obvious that very similar distributions were to be expected if one of these objects could be observed in a direction normal to the line of vision. For instance, seen in such a direction from the northwest, the Cygnus loop could also not show a large positive velocity, since there is little luminous matter in the southeast quadrant.

If the velocity of expansion of $116 \mathrm{~km} / \mathrm{sec}$ for NGC 6960 and NGC 6992 is combined with the motion of $0.03^{\prime \prime}$ per year, the distance of the Cygnus loop is found to be $770 \mathrm{pc}$. This distance leads to a diameter of $40 \mathrm{pc}$. The scanty observations of IC 443 suggest that it is an object of similar size but at a substantially larger distance. The motion at the edge is observable, but it is much smaller than in the Cygnus loop-of the order of $0.01^{\prime \prime}$ per year-and the time interval available is still too small for exact measurement. The velocity of expansion also seems to be comparable to that of the Cygnus loop.

In none of these objects can a star be found that might be responsible for the excitation of the gas. Indeed, Oort's suggestion implies that the collision of the expanding mass with the interstellar gas supplies the excitation. An observational decision on the type of excitation can be obtained from a study of the relative intensities of the emission lines. Since the forbidden lines are always excited by collisions their relative intensities cannot furnish any clues as to whether the excitation is due to ultraviolet radiation from a hot star or to heating of the gas by dissipation of mechanical energy. The intensities of the Balmer lines relative to the forbidden lines depend on the type of excitation, but the interpretation requires knowledge of the state of ionization and of the chemical abundances. The same is true for the relative intensities of permitted lines such as those of $\mathrm{H}$ and $\mathrm{He}$.

The best way to a decision is probably a study of the Balmer decrement. Conditions similar to those expected for collisional excitation can arise with certain types of optical excitation, such as Case $\mathrm{C}$ of Menzel, ${ }^{7}$ excitation of gas optically thin for the exciting radiation by a star which has a blackbody spectrum

\footnotetext{
${ }^{7}$ Menzel, Baker, and Aller, Astrophys. J. 88, 422 (1938).
}

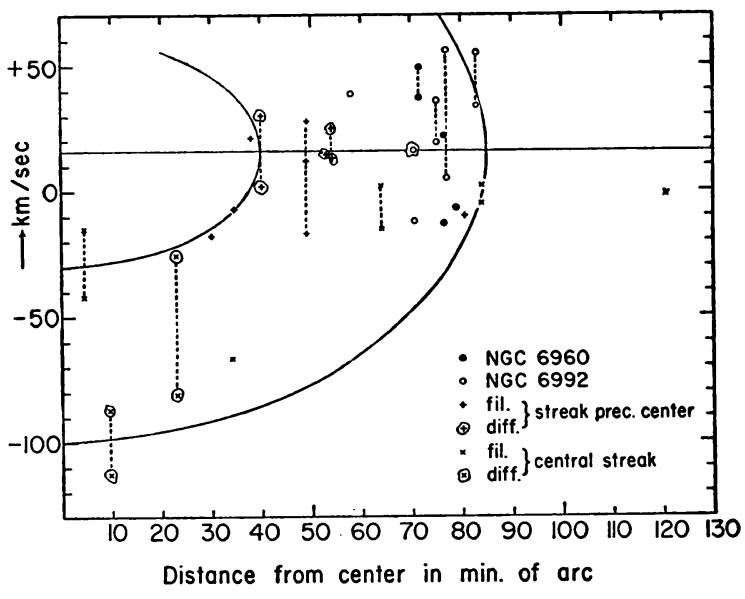

FIG. 1. Radial velocities in the Cygnus loop. Dashed lines connect velocities of different features observed on one spectrogram with a slit length of $30^{\prime \prime}$.

in the Lyman- $\alpha$ region. Cases of this type, however, seem rather unrealistic and, if they are disregarded, the intensity ratio $\mathrm{H} \alpha / \mathrm{H} \beta$ provides a clearcut decision. If this intensity ratio is larger than 3 , collisional excitation is by far the most probable type of excitation.

The pertinent values of the line intensities are in Table I. The results by Chamberlain ${ }^{8}$ were obtained from near monochromatic direct photographs with the 24-in. Schmidt telescope of the University of Michigan. His value for the ratio of the [OII] doublet $\lambda 3727$ to [OIII] $\lambda(4959 / 5007$. agreed very well with that derived from slit spectrograms ${ }^{9}$ which had been obtained earlier by Humason and which, by a fortunate accident, could be calibrated with the aid of step exposures of planetary nebulae obtained by K. Seyffert with the same spectrograph and the same photographic emulsion. It seemed justified to combine the results. The low value of 1.86 for the ratio $(\mathrm{H} \alpha+[\mathrm{NII}]) / \mathrm{H} \beta$ which was obtained in this way seemed to rule out completely the possibility of collisional excitation.

It became clear that this result with its implications, which in view of the absence of an observable exciting star are rather unpleasant, was not definitive when Pickelner ${ }^{10}$ from observations with the nebular spectrograph of the Simeis Observatory found a ratio 6.1 of $(\mathrm{H} \alpha+[\mathrm{NII}]) / \mathrm{H} \beta$ which seemed large enough to establish collisional excitation unless the [NII] lines were unusually strong. However, the intensity of the [OII] doublet relative to the [OIII] lines did not agree with the results of Chamberlain and of the author. Thus, further observations were necessary to settle the case. Unpublished observations by A. D. Code with a photoelectric scanning spectrograph attached at the 100-in. telescope on Mount Wilson fully confirmed

${ }^{8}$ J. W. Chamberlain, Astrophys. J. 117, 399 (1953).

9 R. Minkowski, Gas Dynamics of Cosmic Clouds, edited by H. C. van de Hulst and J. M. Burgers (North-Holland Publishing Company, Amsterdam, 1955), p. 106.

${ }_{10}$ S. B. Pickelner, Izvest. Crimean Astrophys. Obs. 12, 93 (1954). 
the results of Chamberlain and of the author in the blue part of the spectrum. A comparison of the lines in the blue-green and in the red has now been carried out with the nebular spectrograph at the 200-in. telescope. The results fully confirm the ratio 6.1 of $(\mathrm{H} \alpha+[\mathrm{NII}]) / \mathrm{H} \beta$ found by Pickelner. However, the intensity of the [NII] lines is considerable and higher than preliminary results obtained earlier had suggested. ${ }^{9}$ The true ratio of $\mathrm{H} \alpha / \mathrm{H} \beta$ therefore is 3.4.

This value definitely favors collisional excitation. It agrees with the value computed by Chamberlain for an electron temperature of $30000^{\circ}$ if the gas is optically thin in the Lyman lines or an electron temperature of more than $80000^{\circ}$ if the gas is optically thick. The accuracy with which the intensity ratio has been established is not very high, but there seems to be no doubt that it corresponds to a high temperature and that it is not inconsistent with the temperature higher than $80000^{\circ}$ which follows from the observed ratio, 10.6 , of the [OIII] lines $(4959+5007) / 4363 .{ }^{9}$ There are actually very large local variations of all relative intensities, and it may be premature to attempt a detailed discussion based on mean values which do not always include the same points of the object. The new values of the line intensities will lead in any case to some changes in the discussions by Pickelner and by Miyamoto ${ }^{11}$ who found that the line intensities favor collisional excitation. But, there is no reason to believe that this conclusion will be changed.

The conclusion that the excitation is collisional finds some support in the very low relative intensity which preliminary observations seem to show for HeI $\lambda 5875$.

Since there seems to be no doubt left that the excitation is collisional, it seems useful to discuss some consequences of the assumption that objects like the Cygnus loop are decelerated shells of originally high velocity of expansion. If such objects are created continuously, their size distribution would show considerable preference for large diameters since the velocity of expansion will decrease with increasing size and the number of objects of a given size will be inversely proportional to the velocity of expansion reached at that size. It seems possible that this may explain at least partly the curious fact that there are no small objects known which belong definitely to this class of objects. There are very few objects which may have to be considered as smaller examples, but they are still fairly sizable, such as NGC $6888,11 \times 16^{\prime}$, which seems to be excited by a Wolf-Rayet star and might therefore not belong in this group, or S $22^{4}$, $10 \times 11^{\prime}$, and a very similar nebula, $9 \times 12^{\prime}$ at (1950) $7^{h} 26^{m} 15^{s},+13^{\circ} 21^{\prime}$. In any case, it does not seem possible at this time to trace observationally the history of objects of this class.

For a preliminary exploration of the conditions under which such objects might be formed it seems

\footnotetext{
11 S. Miyamoto, Z. Astrophys. 38, 245 (1956).
}

adequate to carry out the simplified discussion by Oort in somewhat more detail. Let us assume that the shell is being retarded just by sweeping up the interstellar material which it meets. If other effects, such as compression of the material in front of the shell or radiation losses would contribute to the deceleration, we would overestimate the interstellar density needed to cause a given retardation. If $M_{0}$ is the original mass of the shell, $v_{0}$ the original velocity of ejection, $r_{1}$ its present radius, $v_{1}$ the present velocity of expansion, and $\rho$ the density of the interstellar material, we have

$$
M_{0} v_{0}=\left(M_{0}+\frac{4 \pi}{3} r_{1}^{3} \rho\right) .
$$

It is sufficient to consider the late stages of development where $M_{0}$ is small compared to the mass of the swept-up shell. In this case we obtain, expressing $M_{0}$ in solar units, $v_{0}$ and $v_{1}$ in $\mathrm{km} / \mathrm{sec}, r_{1}$ in parsecs, and replacing $\rho$ by the density $\mathrm{N}_{\mathrm{H}}$ in $\mathrm{H}$ atoms per $\mathrm{cm}^{3}$,

$$
M_{0} v_{0}=0.1 N_{\mathrm{H}} r_{1}^{3} v_{1}
$$

or, for the Cygnus loop with $r_{1}=20 \mathrm{pc}$ and $v_{1}=100$ $\mathrm{km} / \mathrm{sec}$,

$$
M_{0} v_{0}=8 \times 10^{4} N_{\mathrm{H}} .
$$

It is at once evident that, unless we want to admit an absurdly low interstellar density, we cannot assume that the original object was an ordinary nova or a planetary nebula for which $M_{0} v_{0}$ is of the order $10^{-1}$ or 1 , respectively; the required interstellar densities of the order $10^{-6}$ or $10^{-5} \mathrm{~cm}^{-3}$ are much too low. For a supernova of type I, such as the Crab Nebula, $M_{0} v_{0}$ is of the order $10^{2}$; the required interstellar density of $10^{-3} \mathrm{~cm}^{-3}$ still is not plausible. We do not know the mass ejected by a supernova of type II, but the initial velocity seems to be of the order of $5000 \mathrm{~km} / \mathrm{sec}$, or even higher. Since the supernovae of type II belong to Baade's population I, they may be massive stars and it is not inconceivable that the ejected mass is of the order 1, ten times larger than for a supernova of type I. If then we assume $M_{0} v_{0}$ to be of the order $5 \times 10^{3}$, the required interstellar density would be 0.06 , still a low, but not unacceptable value. From $M_{0}=1, v_{0}=5000$, $v_{1}=100$ it follows directly that the present mass is 50 , if the shell is still complete.

The actual mass of the Cygnus loop is not known, but it must be large. If the distance is $770 \mathrm{pc}$, a typical bright filament will have a diameter of the order $0.01 \mathrm{pc}$ and a length of the order of $1 \mathrm{pc}$. The electron density of such filaments is 500 to $1000 \mathrm{~cm}^{-2}$ according to unpublished measures by Osterbrock of the intensity ratio of the components of the [OII] doublet. The mass of a single bright filament is thus of the order 0.01 . Since there is a large number of filaments and in addition diffuse masses, it seems reasonable to think 
of a mass of one or several solar masses. ${ }^{12}$ Since the Cygnus loop is obviously far from being a complete shell, such a value would not be inconsistent with the mass 50 expected for a complete shell.

We may now ask whether under our assumptions the appearance of the Cygnus loop and of IC 443 as imcomplete thick shells can be reasonably interpreted. To obtain this appearance it is obviously not necessary to find a thick layer of matter if one proceeds in any direction from the center of expansion. It is only necessary that in different directions, matter is found at different distances. This situation would occur if the interstellar matter were inhomogeneous, and it is indeed likely that interstellar densities vary over such distances as 40 pc. From (3) we find the interstellar density to be inversely proportional to $r_{1}^{3} v_{1}$. For the outer border we have $r_{1}=20 \mathrm{pc}, v_{1}=116 \mathrm{~km} / \mathrm{sec}$ and for the inner border $r_{1}=10 \mathrm{pc}, v_{1}=46 \mathrm{~km} / \mathrm{sec}$. The density encountered by the matter now at the inner border must thus have been about 20 times higher than the value of 0.06 found for the outer border, or about $1.2 \mathrm{~cm}^{-3}$. The range of densities which is required seems entirely reasonable.

The proposed interpretation of the appearance of the Cygnus loop and of IC 443 may perhaps lead to an explanation of the curious fact found by Rishbeth ${ }^{13}$ that the nonthermal radio source resembles in the distribution of surface brightness the nebula whose line emission seems to be of thermal origin. If at this moment the bright matter at the northeast edge of the nebula encounters interstellar matter of particularly high density, compression of matter may lead to strong optical emission by heating, and compression of magnetic fields may lead to strong radio emission.

12 Note added in proof.-A somewhat smaller mass is suggested by the final results by D. E. Osterbrook, Publs. Astron. Soc. Pacific 70, 180 (1958).

${ }^{13}$ H. Rishbeth, Australian J. Phys. 9, 494 (1956).
Finally, we may discuss the question of the age and the frequency of these objects. Integration of (2) leads to

$$
r=8 \times 10^{-2}\left(v_{0} M_{0} t / n_{\mathrm{H}}\right)^{4},
$$

where the time is in years. For the Cygnus loop we obtain an age of $47000 \mathrm{yr}$.

In order to obtain the frequency of such objects on the assumption that they originate from supernovae of type II, we have to know the age at which such an object disappears. This is difficult to estimate, but it is perhaps reasonable to assume that this occurs when the velocity of expansion becomes comparable to random velocities, a stage which has almost been reached in the Cygnus loop. From (2) and (4) we find that the velocity of expansion will decrease as $t^{-}$. This leads to the expectation that the total lifetime of the Cygnus loop is of the order of $100000 \mathrm{yr}$. The Cygnus loop may have been formed in a region of somewhat less than average density. The average life time thus might be shorter, but hardly by a large factor. Since supernovae of type II seem to occur with a frequency of one supernova per galaxy per $50 \mathrm{yr}$, we are led to expect that the number of objects of this type in the Galaxy is of the order 1000. This number seems rather large compared to the number of 5 or perhaps 8 known objects of this type, particularly since the frequency of members of population I should not increase strongly in the central parts of the galaxy. But, in view of the crudity of this discussion and of some of the data on which it is based, the discordance does not appear serious.

At the present stage it seems that supernovae of type II are the only known objects which may lead to the formation of such objects as the Cygnus loop. The assumption that they are actually the origin of this type of object seems to be compatible with all known facts. A more erudite discussion is highly desirable as a basis for a final decision, and a broader observational material is also needed.

\section{DISCUSSION}

V. A. Ambartsumian, Burakan Astrophysical $O b$ servatory, Academy of Sciences of the Armenian S.S.R., Erevan, U.S.S.R.: Do we have any observations on radial velocities in other objects?

R. MINKowSKI, Mount Wilson and Palomar Observtories, Pasadena, California: No, not yet. Such observations require very much time, because the objects are so faint.

J. W. Chamberlain, Yerkes Observatory, Williams Bay, Wisconsin: I do not think that there can be any doubt that the relative line intensities measured by Pickelner and Minkowski from spectra are superior to the relative intensities obtained from direct photo- graphs. It was to be expected that there might very well be systematic errors of the order of a factor of 2 in the relative intensities obtained from filter photographs. While such an error invalidates those data for measurements of the Balmer decrement, the electron densities obtained from them should be sufficiently reliable. I might also mention that the ratio $\mathrm{H} \alpha / \mathrm{H} \beta<1.86$ that Minkowski quoted at the second symposium, which used the relative intensities from filter photographs, is not compatible even with radiative excitation. Although the relative intensities that the Baker-Menzel theory predicts will have to be revised when account is taken of the fine structure, the correction will probably steepen the theoretical Balmer decrement. 
M. J. Seaton, Department of Physics, University College, London, England: I would like to comment on Minkowski's discussion of the $\mathrm{H} \alpha / \mathrm{H} \beta$ ratio. It is sometimes overlooked that the calculations of Baker and Menzel do not represent an exact solution for the radiative Balmer decrements. The quantum states $n l m$ of the hydrogen atom are degenerate with respect to $l$ and $m$, the energy depending only on $n$. The assumption made by Baker and Menzel is that, for given $n$, the populations of the quantum states are the same for all $l$ and $m$. This assumption may be justified for conditions of thermodynamic equilibrium, but may not be true when the excited levels are populated only by capture and cascading. Some preliminary calculations were carried out in Paris two years ago by Claude Joslet. These calculations were intended only as an exploratory survey and the results obtained were, therefore, not published. It was found, however, that the calculated Balmer decrements might be significantly different when proper allowance was made for the individual quantum states. The whole problem is at present being studied in detail by Alan Burgess of University College, London. Until this work is completed, I would hesitate to draw any definite conclusions from observations of the $\mathrm{H} \alpha / \mathrm{H} \beta$ ratios.

F. DRAKE, Harvard College Observatory, Cambridge, Massachusetts: I would like to mention some radio observations that are relevant to this discussion. We have observed the $21-\mathrm{cm}$ line in the direction of the Cygnus loop and find that the hydrogen density is very low there, probably consistent with the density which is called for by Minkowski's interpretation. We also have observations in the direction of Simeis 147, and in this position, we find very marked evidence for an expanding shell of neutral hydrogen. If such is the case, the shell has an expansion velocity of $12 \mathrm{~km} / \mathrm{sec}$ and an extremely small velocity dispersion of the order of 1 or $2 \mathrm{~km} / \mathrm{sec}$. This is similar in some respects to the shells that we have observed in other objects such as the Orion Nebula region and in Lambda Orionis. One would expect in time that a shell expansion velocity would decrease and the velocity dispersion would increase, so that the shell connected with Simeis 147 would eventually become of the form we observe in the Orion Nebula region. Because of these observations, it would seem possible that there is some connection between the Cygnus loop structures and these older structures, and it seems possible that the Cygnus loop structures may be a young stage of the Orion-type structures.

M. P. SAVEDOFF, Department of A stronomy, University of Rochester, Rochester, New York: I noticed two numbers that struck me as different. First, the figure of 1 supernova per $50 \mathrm{yr}$. Secondly, the mass of the Crab is still quoted as $0.1 \mathrm{M} \odot$; that mass was based, I believe, on calculations before the cyclotron radiation explanation was given. Do you still believe that figure?

R. MINKOWSKI : As regards the supernovae frequency, there are two types of supernovae. The supernovae I seem to occur about every 400 or 500 years per galaxy, and the supernovae II about every 50 years per galaxy, with considerable leeway. But the supernovae II are certainly much more frequent than the supernovae I. A mass of the Crab Nebula of the order of $0.1 \mathrm{M} \odot$ comes now not only from theoretical discussions by Pickelner and Shklovsky and by others, but also from unpublished measurements of electron densities in the filaments of the Crab Nebula by Osterbrock, who found that the integrated mass of the filaments is of the order of $0.1-0.2 \mathrm{M} \odot$. This order of magnitude of the mass of the Crab Nebula seems now to be fairly definite. 